\title{
Targeted Sequencing Facilitated Diagnosis of an Uncommon Patient Harboring Both Multiple Primary and Intrapulmonary Metastatic Lung Cancer: A Case Report
}

\author{
Hefei Li \\ Shaoyong Dong' \\ Duo Zhang' \\ Zhimin Guo' \\ $\mathrm{Ce} \mathrm{Li}$ \\ Jianxing Xiang ${ }^{2}$ \\ Xiao Zou ${ }^{2}$ \\ Li Yan ${ }^{2}$ \\ Ying Sun ${ }^{2}$ \\ Wei $\mathrm{Li}^{1}$ \\ 'Department of Thoracic Surgery, \\ Affiliated Hospital of Hebei University, \\ Baoding, 071000, People's Republic of \\ China; ' ${ }^{2}$ Department of Medicine, Burning \\ Rock Biotech, Guangzhou, 510300, \\ People's Republic of China
}

\begin{abstract}
Estimated to comprise approximately $10 \%$ of lung cancer cases, multiple pulmonary lesions pose a diagnostic and therapeutic challenge in thoracic oncology. Distinction between multiple primary lung cancer (MPLC) and intrapulmonary metastasis (IPM) directly affects tumor staging and clinical management. In equivocal cases in which the lesions are histopathologically indistinguishable, targeted sequencing can provide key additional evidence for differential diagnosis. Herein, we describe an unusual patient who presented with seven lung lesions that consisted of primary tumors and metastatic lesions, each showing distinct clonality status based on histomolecular findings. Specifically, the 45 -year-old female never-smoker underwent a surgery that removed one invasive lepidic predominant adenocarcinoma and five microinvasive adenocarcinomas. Next-generation sequencing revealed three of the lesions to carry a clonal driver mutation EGFR p.L858R, supporting an IMP diagnosis. EGFR p.L858R was not detected in two other surgical specimens, which instead harbored respective oncogenic $B R A F$ p.G469A and an uncommon EGFR p.G779F. These results led to diagnosis of the two lesions as primary tumors of lineages different from that of the metastases. The patient had achieved a recurrence-free survival of 21 months as of the latest follow-up. In this rare case that presented with evidence of both MPLC and IPM, targeted sequencing proved valuable in facilitating the diagnostic workup.
\end{abstract}

Keywords: multiple primary lung cancer, intrapulmonary metastasis, targeted sequencing, mutational profiling, clonality

\section{Introduction}

Sensitive lung cancer detection enabled by advanced computed tomography technology has increasingly identified patients presenting with multiple lung lesions. Estimated to represent $\sim 10 \%$ of lung cancer cases, multiple pulmonary lesions pose a diagnostic and therapeutic challenge in thoracic oncology. ${ }^{1}$ A definitive diagnosis as multiple primary lung cancer (MPLC) or intrapulmonary metastasis (IPM) relates directly to tumor staging and subsequent management. Criteria for MPLC diagnosis were first proposed 45 years ago. $^{2}$ In 2016, the International Association for the Study of Lung Cancer (IASLC) proposed an updated system that integrated clinical, histopathological, and oncogenomic approaches. ${ }^{3}$ Specifically, this comprehensive diagnostic workup relies on characterizing intrinsic nature of the lesions including rate of expansion, histological subtype, pathological biomarkers, and clonal genomic alterations. In difficult cases in
Correspondence: Wei Li

Department of Thoracic Surgery,

Affiliated Hospital of Hebei University,

Baoding, 071000, People's Republic of

China

Tel $+86312-5981670$

Email liweil5930923618@I63.com 
which the lesions are histopathologically indistinguishable or highly similar, the differential diagnosis is facilitated by molecular profiling tools such as comparative genomic hybridization and mutational analysis with polymerase chain reaction or next-generation sequencing (NGS). ${ }^{4}$

Of these available methods, NGS offers a unique combination of advantages. In addition to the ability to detect multiple types of genomic aberrations with high throughput, NGS has already been integrated into routine clinical oncology practice, especially in that of non-small cell lung cancer (NSCLC). Herein, we describe an unusual patient who presented with six lung lesions, of which at least one was diagnosed to have resulted from IPM and two to be distinct primary tumors based on histopathology and NGS profiling.

\section{Case Report}

Multiple small space-occupying lesions were noted in a 45-year-old female never-smoker during a chest computed tomography (CT) scan in Jan. 2019 (Figure 1). There was no remarkable change in lesion size during the four follow-up CT examination prior to surgical removal. In February 2019, the patient received singleport VATS right upper lobectomy, wedge resection of the right lower lobe, and lymphadenectomy, although masses in the left upper and lower lobes were not excised due to their small size. Histopathological review of the six surgical specimens indicated one invasive lepidic predominant adenocarcinoma (LPA) and five microinvasive adenocarcinomas (MIAs, Figure 2). No nodal metastasis was noted.
Next-generation sequencing with a targeted panel of 168 cancer-related genes (Burning Rock Biotech, Guangzhou, China) was performed on all six samples. A summary of the histopathological and molecular analyses is listed in Table 1 . Specimen 1 was the only invasive LPA lesion, but it showed a mutation profile highly concordant with that of lesion 2, sharing oncogenic EGFR p.L858R and two other uncharacterized alterations to $S T K 11 / L K B 1$. Lesion 2 also shared histological subtype and $E G F R$ p.L858R with lesion 5, although the latter harbored a unique $R B M 10 \mathrm{p}$. G811fs frameshift mutation. Additionally, EGFR p.G779F and $B R A F$ p.G469A were detected in lesions 3 and 4, respectively, and no genomic aberration associated with the 168 interrogated genes was identified in lesion 6 . Based on the histological class and oncogenic driver EGFR p.L858R shared by specimens 5 and 2, as well as highly similar histomolecular phenotypes between specimens 1 and 2, clonality was established between among the three lesions, thereby indicating the presence of metastases. On the other hand, despite the same histology, absence of EGFR p.L858R in samples 3 and 4 provided evidence for primary tumors that evolved in parallel with lesions 1 and 2. Therefore, the patient was diagnosed with both intrapulmonary metastatic and primary lesions by histomolecular characterization. As of the latest followup in Nov. 2020, the patient had shown no evidence of disease, thereby achieving a recurrence-free survival of 21 months.

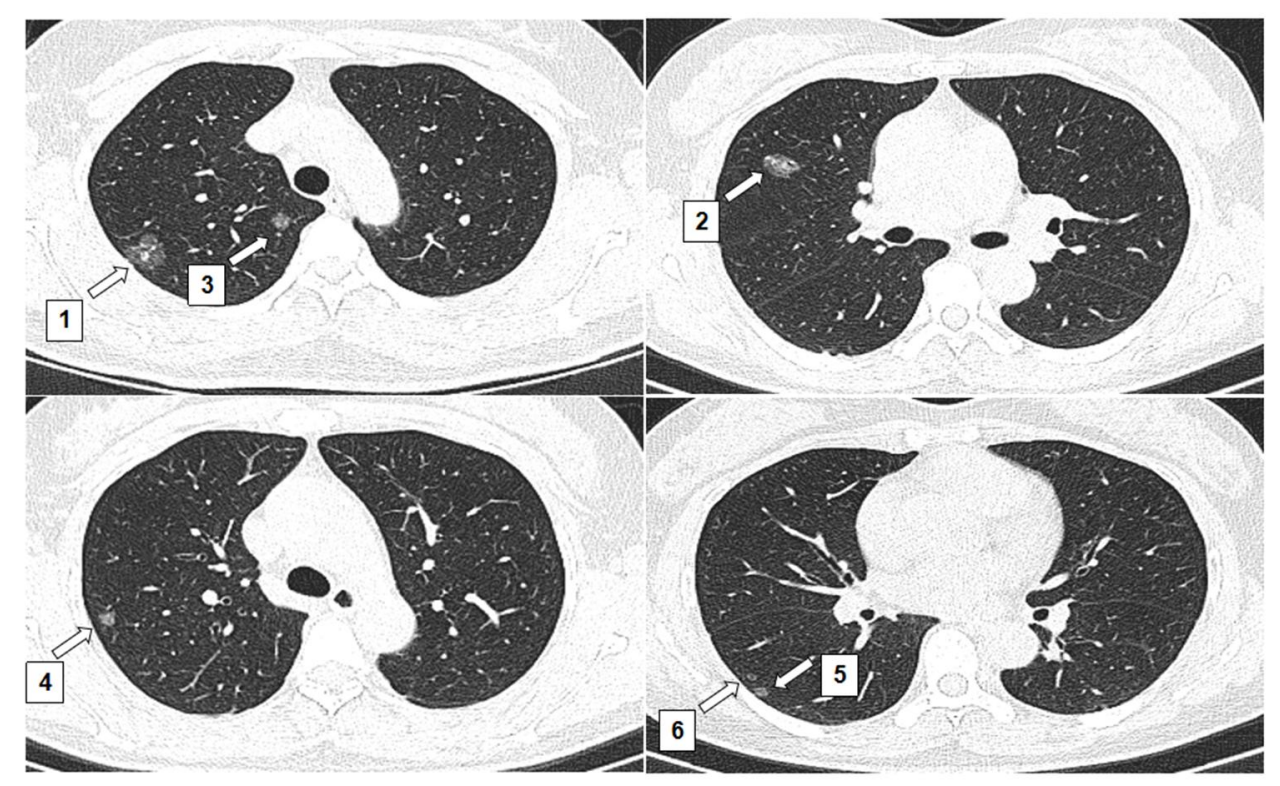

Figure I Chest computed tomography (CT) showed multiple space-occupying lesions. The arrows indicate the lesions. 

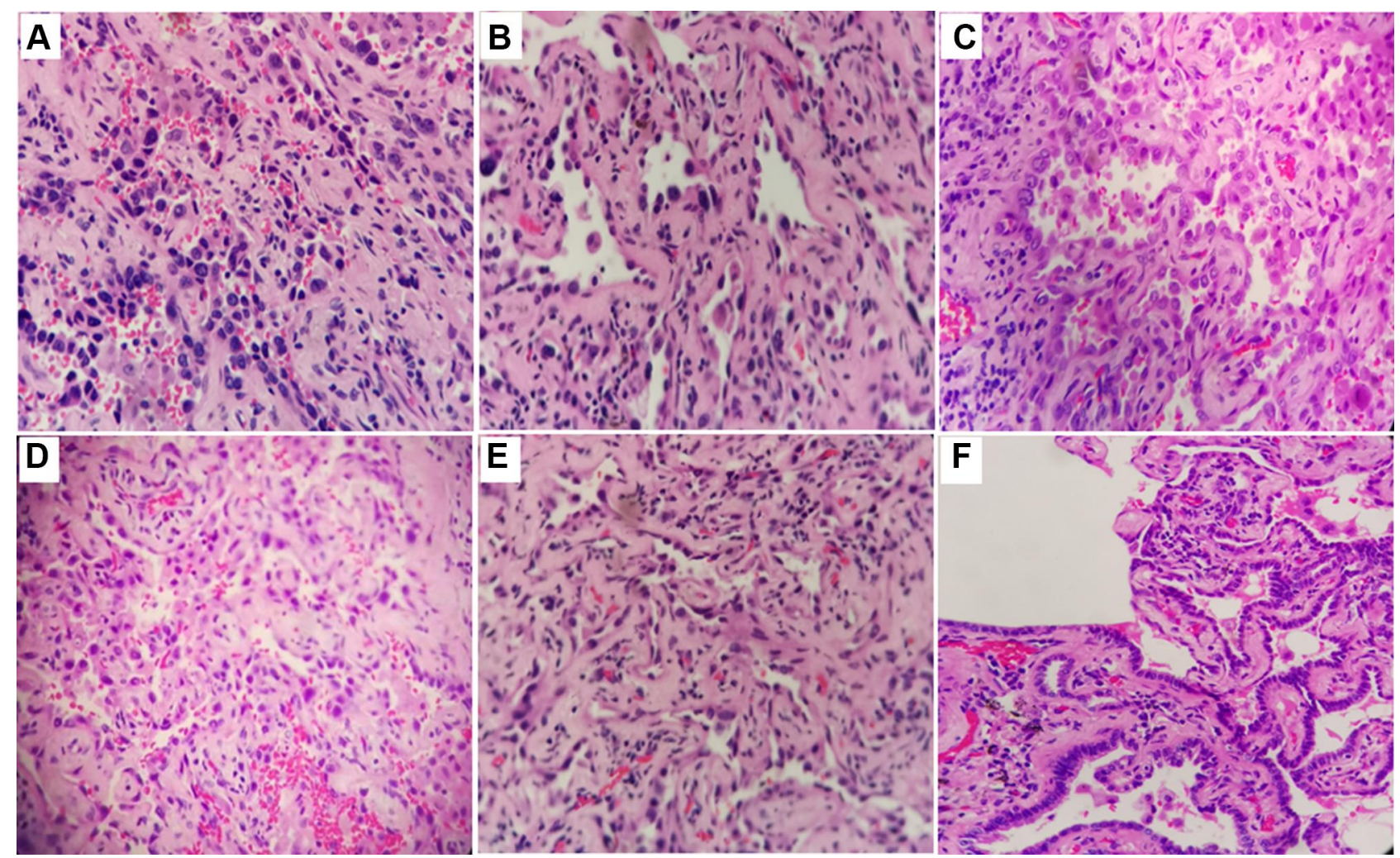

Figure 2 Results from histopathological examination of the multiple space-occupying lesions. (A) Invasive lepidic predominant adenocarcinoma for lesion I; (B) microinvasive adenocarcinoma for lesion 2; (C) microinvasive adenocarcinoma for lesion 3; (D) microinvasive adenocarcinoma for lesion 4; (E) microinvasive adenocarcinoma for lesion 5; (F) microinvasive adenocarcinoma for lesion 6 .

\section{Discussion}

Diagnosis and management of MPLC remains controversial, as currently there is no robust diagnostic workup capable of making clear distinction between multiple primary and metastatic disease. Instead, comprehensive synthesis of multidisciplinary evidence is recommended, which leaves room for judgement calls in equivocal cases. When differences in size, growth rate, and histology of multiple lesions are too small to justify a definitive diagnosis, profiling of genomic alterations could reveal the clonality status of these lesions. The TRACERx project tracked the evolution of NSCLC in early-stage patients and found that driver mutations in EGFR, MET, BRAF, and TP53 were almost always clonal. ${ }^{5}$ Another study compared the mutations in EGFR, $K R A S, A L K$ and $B R A F$ between primary tumors and metastases in 25 lung cancer patients, and mutational concordance reached $96 \% .{ }^{6}$ In particular, EGFR mutations are rarely heterogeneously distributed in lung

Table I Clinicopathological Characteristics and Mutation Profiles of the Surgical Resections

\begin{tabular}{|c|c|c|c|c|c|c|c|c|c|c|}
\hline \multirow{2}{*}{$\begin{array}{l}\text { Lesion } \\
\text { Number }\end{array}$} & \multirow[t]{2}{*}{ Site } & \multirow{2}{*}{$\begin{array}{l}\text { Size } \\
(\mathrm{cm})\end{array}$} & \multirow{2}{*}{$\begin{array}{c}\text { Predominant } \\
\text { Pattern }\end{array}$} & \multicolumn{7}{|c|}{ Detected Mutations } \\
\hline & & & & $\begin{array}{c}\text { EGFR p. } \\
\text { L858R }\end{array}$ & $\begin{array}{c}\text { EGFR p. } \\
\text { G779F }\end{array}$ & $\begin{array}{c}\text { BRAF p. } \\
\text { G469A }\end{array}$ & $\begin{array}{c}\text { RBMIO } \\
\text { p.G8 I Ifs }\end{array}$ & $\begin{array}{c}\text { ERBB2 } \\
\text { p.L755A }\end{array}$ & $\begin{array}{c}\text { STKII } \\
\text { E35I_D352del }\end{array}$ & $\begin{array}{l}\text { STKII- } \\
\text { ELAVLI } \\
\text { Fusion }\end{array}$ \\
\hline 1 & RUL & 1.6 & LPA & + & \multirow{6}{*}{+} & \multirow{6}{*}{+} & \multirow{6}{*}{+} & \multirow{6}{*}{+} & \multirow{6}{*}{$\begin{array}{l}+ \\
+\end{array}$} & \multirow{6}{*}{$\begin{array}{l}+ \\
+\end{array}$} \\
\hline 2 & RUL & 1.2 & MIA & + & & & & & & \\
\hline 3 & RUL & 0.6 & MIA & & & & & & & \\
\hline 4 & RUL & 0.5 & MIA & \multirow{3}{*}{+} & & & & & & \\
\hline 5 & RLL & 0.5 & MIA & & & & & & & \\
\hline 6 & RLL & 0.2 & MIA & & & & & & & \\
\hline
\end{tabular}

Abbreviations: LPA, lepidic predominant adenocarcinoma; MIA, microinvasive adenocarcinoma; RLL, right lower lobe; RUL, right upper lobe. 
adenocarcinoma. ${ }^{7}$ In the present case, lesions 1,2 , and 5 all harbored EGFR p.L858R, a known sensitizing mutation susceptible to therapeutic intervention with EGFR TKIs. More importantly, this driver mutation also pointed to shared clonality of the three lesions. It is less clear which were the metastases, although the higher concordance of mutation profiles between samples 1 and 2 suggested closer evolutionary relationship. Also, as the $R M B 10 \mathrm{p} .811 \mathrm{fs}$ mutation in lesion 5 may be a passenger mutation, it is also possible that all three were metastases that had evolved from a common lineage. Regardless of the exact evolutionary history, there was at least one metastasis among lesions 1 and 2. On the other hand, lesions 3 and 4 demonstrated non-overlapping mutation profiles with lesions 1 and 2 . Moreover, sample 3 carried $B R A F$ p.G469A, an oncogenic $B R A F$ hotspot mutation. ${ }^{8}$ Based on previous findings of the clonality of $E G F R$ and $B R A F$ driver alterations, lesions 3 and 4 were deemed as primary tumors separate from the lineage of lesions 1, 2 and 5. Thus, histomolecular characterization of the multiple lesions in this case led to a diagnosis of co-existing MPLC and IPM. However, there is still no unified standard for differentiating MPLC and IPM, and more prospective clinical trials are needed. Thus, this case is a highly uncommon one that features concurrent MPLC and IMP. There have been a number of studies justifying surgery in treating MPLC or proposing algorithms to differentiate between MPLC and IPM. However, the majority included patients with either MPLC or IMP. Additionally, although surgery plus adjuvant chemotherapy is recommended by certain guidelines, in this case chemotherapy was not administered and achieved an encouraging recurrence-free survival of 21 months as of latest follow-up. Therefore, this report can enrich the knowledge regarding the diagnosis, treatment, and clinical outcome of patients with this unusual condition and serve as a reference for similar future cases.

\section{Conclusion}

In summary, our case demonstrated the efficacy of targeted sequencing in distinguishing MPLC from IPM in diagnosing patients with histopathologically similar multiple lung lesions. Targeted sequencing can therefore be useful in enhancing the diagnostic process.

\section{Abbreviations}

NSCLC, non-small cell lung cancer; NGS, next-generation sequencing; MPLC, multiple primary lung cancer; IPM, intrapulmonary metastasis; $\mathrm{CT}$, computed tomography; LPA, lepidic predominant adenocarcinoma; MIA, microinvasive adenocarcinoma.

\section{Ethical Approval}

Institutional approval was not required to publish the case details.

\section{Consent for Publication}

Written informed consent was obtained from the patient for use of her samples, images and the publication of this report.

\section{Acknowledgments}

The authors sincerely thank the patient and her family.

\section{Disclosure}

The authors report no conflicts of interest in this work.

\section{References}

1. Mansuet-Lupo A, Barritault M, Alifano M, et al. Proposal for a combined histomolecular algorithm to distinguish multiple primary adenocarcinomas from intrapulmonary metastasis in patients with multiple lung tumors. $J$ Thorac Oncol. 2019;14:844-856. doi:10.1016/j.jtho.2019.01.017

2. Martini N, Melamed MR. Multiple primary lung cancers. J Thorac Cardiovasc Surg. 1975;70:606-612. doi:10.1016/S0022-5223(19) 40289-4

3. Detterbeck FC, Franklin WA, Nicholson AG, et al. The IASLC lung cancer staging project: background data and proposed criteria to distinguish separate primary lung cancers from metastatic foci in patients with two lung tumors in the forthcoming eighth edition of the TNM classification for lung cancer. $J$ Thorac Oncol. 2016;11:651-665. doi:10.1016/j.jtho.2016.01.025

4. Schneider F, Dacic S. Histopathologic and molecular approach to staging of multiple lung nodules. Transl Lung Cancer Res. 2017;6:540-549. doi:10.21037/tlcr.2017.06.11

5. Jamal-Hanjani M, Wilson GA, McGranahan N, et al. Tracking the evolution of non-small-cell lung cancer. $N$ Engl $J$ Med. 2017;376:2109-2121. doi:10.1056/NEJMoa1616288

6. Asmar R, Sonett JR, Singh G, Mansukhani MM, Borczuk AC. Use of oncogenic driver mutations in staging of multiple primary lung carcinomas: a single-center experience. $J$ Thorac Oncol. 2017;12:1524-1535. doi:10.1016/j.jtho.2017.06.012

7. Yatabe Y, Matsuo K, Mitsudomi T. Heterogeneous distribution of EGFR mutations is extremely rare in lung adenocarcinoma. $J$ Clin Oncol. 2011;29:2972-2977. doi:10.1200/JCO.2010.33.3906

8. Virzi AR, Gentile A, Benvenuti S, Comoglio PM. Reviving oncogenic addiction to MET bypassed by BRAF (G469A) mutation. Proc Natl Acad Sci U S A. 2018;115:10058-10063. doi:10.1073/ pnas. 1721147115 


\section{Publish your work in this journal}

OncoTargets and Therapy is an international, peer-reviewed, open access journal focusing on the pathological basis of all cancers, potential targets for therapy and treatment protocols employed to improve the management of cancer patients. The journal also focuses on the impact of management programs and new therapeutic

Submit your manuscript here: https://www.dovepress.com/oncotargets-and-therapy-journal agents and protocols on patient perspectives such as quality of life, adherence and satisfaction. The manuscript management system is completely online and includes a very quick and fair peer-review system, which is all easy to use. Visit http://www.dovepress.com/ testimonials.php to read real quotes from published authors. 